\title{
A Scale-Space Approach for Image Registration of Vessel Structures
}

\author{
Stefan Heldmann, Nils Papenberg \\ Institute of Mathematics, University of Lübeck \\ heldmann@math. uni-luebeck.de
}

\begin{abstract}
We present a multi-scale approach for non-rigid image registration of tubular tree-like structures such as vessels. Therefore, we consider a Gaussian and a combined morphological and Gaussian scalespace. Similar to a multi-level framework, we solve a sequence of registration problems on several scale-space levels using a standard variational approach for non-liner volumetric image registration. The overall idea is to avoid locally ambiguous mappings between parts of the images by removing morphological details but also finding a global optimal solution by spreading remaining local information using Gaussian scaling. We successfully tested our method on registration of 3D CT and ultrasound images of hepatic vessels.
\end{abstract}

\section{Introduction}

Image registration of tubular tree-like structures is a very difficult task. Our work is motivated by a project on Liver surgery. Here, image registration comes into play for the alignment of $3 \mathrm{D}$ images of liver where the target structures are vessels. Another example is the analysis of lungs where registration is used for the alignment of the respiratory system. The particular difficulty for image registration is that there are locally many ambiguous mappings between tree-like structures which makes it hard to find a globally optimal alignment.

State-of-the-art approaches rely on intelligent pre-processing. Typically, in a first step segmentation is used to extract vessels. Model-based approaches then try to identify few bifurcation points that are used as landmarks $[1,2]$ and/or to build a 3D model from the image data $[3,4,5]$. Other approaches use volumetric image registration techniques focusing on edges in the image data. However, all these approaches suffer from the local structure such that extensive user-interaction by an expert is required typically by identifying corresponding bifurcation points.

Our new approach uses ideas from scale-space. For the alignment of major structures we spread local information in the images and construct a Gaussian scale-space from the images and use it in a multi-level fashion. Furthermore, we extent the Gaussian scale-space by a second morphological scale that builds on the observation that anatomical tree-like structures become thinner as ramification becomes finer. 


\section{Material and methods}

We use a standard variational approach for non-liner volumetric image registration [6]. Given two images $R$ (reference) and $T$ (template) we compute a deformation $y$ of the template by minimizing a suitable cost-function $\mathcal{J}$ given by

$$
\mathcal{J}(R, T, y):=\mathcal{D}(R, T(y))+\mathcal{S}(y)
$$

Here, $\mathcal{D}$ measures the distance between the reference $R$ and the deformed template $T(y)$. The second building block $\mathcal{S}$ penalizes derivatives of the deformation and thus forces smoothness. We assume the images have been preprocessed by segmentation and therefore are binary images. Consequently, we choose the sum-of-squared-difference (SSD) distance measure. We will not discuss a particular regularizer here. Suitable choices include diffusive, elastic, or curvature regularization [6].

Instead of using the original images directly we construct a scale-space [7] from the images by successively smoothing the image data and removing details. Finally, we solve a sequence of registration problems. Starting on the coarsest scale (least detailed images) we compute a deformation and use the result as initial guess for the next finer scale.

We consider two strategies. First, we construct a Gaussian scale by smoothing the original image data and creating an image pyramid for its use in a multi-level framework. This procedure basically spreads local information. Second, we extend the pure Gaussian scale by a morphological axis to eliminate small vessels structures and thus to focus on the alignment of thick vessels. The second approach is a generalization of the first one. Therefore, we only describe the combined morphological and Gaussian approach in the following.

Addressing the problem of locally ambiguous mappings we introduce morphological scaling of the images. The leading idea is that major vessels are thicker than peripheral ones. For that reason, we use a morphological opening with a ball $\bar{B}_{r}:=\{x:\|x\| \leq r\}$ of radius $r$. For binary images $R, T: \mathbb{R}^{d} \rightarrow\{0,1\}$ we set

$$
R^{r}:=\left(R \ominus \bar{B}_{r}\right) \oplus \bar{B}_{r} \quad \text { and } \quad T^{r}:=\left(T \ominus \bar{B}_{r}\right) \oplus \bar{B}_{r}
$$

where $\left(R \ominus \bar{B}_{r}\right)(x):=1$ if $R=1$ on $x+\bar{B}_{r}$ and 0 else denotes erosion and $\left(R \oplus \bar{B}_{r}\right)(x):=1$ if $R \neq 0$ on $x+\bar{B}_{r}$ and 0 else is dilation. As a result, for $r=0$
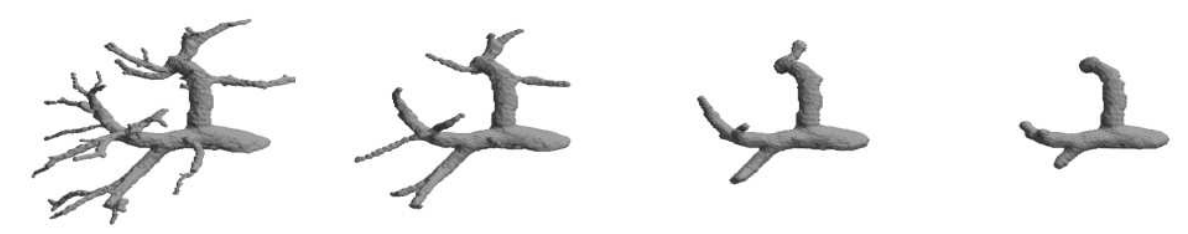

Morphological Scale

Fig. 1. Morphological scale-space of a 3D vessel system (increasing $r$ left to right) 
we have $R^{0}=R, T^{0}=T$ and for increasing scale-space parameter $r$ we remove details from the images. An example is given in Figure 1.

Besides ambiguous mappings another problem is that structural information is very local, i.e., corresponding vessels do not initially overlap which makes registration difficult. To this end, we additionally use a Gaussian scale-space. We define

$$
R^{r, \sigma}:=R^{r} * K_{\sigma} \quad \text { and } \quad T^{r, \sigma}:=T^{r} * K_{\sigma}
$$

where $K_{\sigma}(x)=\left(2 \pi \sigma^{2}\right)^{-\frac{d}{2}} \exp \left(-\frac{\|x\|^{2}}{2 \sigma^{2}}\right)$ is a Gaussian with standard deviation $\sigma$. Consequently, $R^{r, \sigma} \rightarrow R^{r}, T^{r, \sigma} \stackrel{2 \sigma^{2}}{\rightarrow} T^{r}$ as $\sigma \rightarrow 0$ and with increasing $\sigma$ more and more local information is dispersed. A $2 \mathrm{D}$ example for simulated data with combined morphological and Gaussian scale-space is shown in Figure 2.

Summarizing, we solve a sequence of registration problems for scale-space parameters $\left(r_{1}, \sigma_{1}\right), \ldots,\left(r_{N}, \sigma_{N}\right)$ with $r_{1} \geq \ldots \geq r_{N}=0$ and $\sigma_{1} \geq \ldots \geq \sigma_{N}=0$. Note that choosing $r_{1}=\ldots=r_{N}=0$ results a pure Gaussian scale space.

For our numerical method we first discretize the objective function (1) and use a Gauss-Newton optimization method.

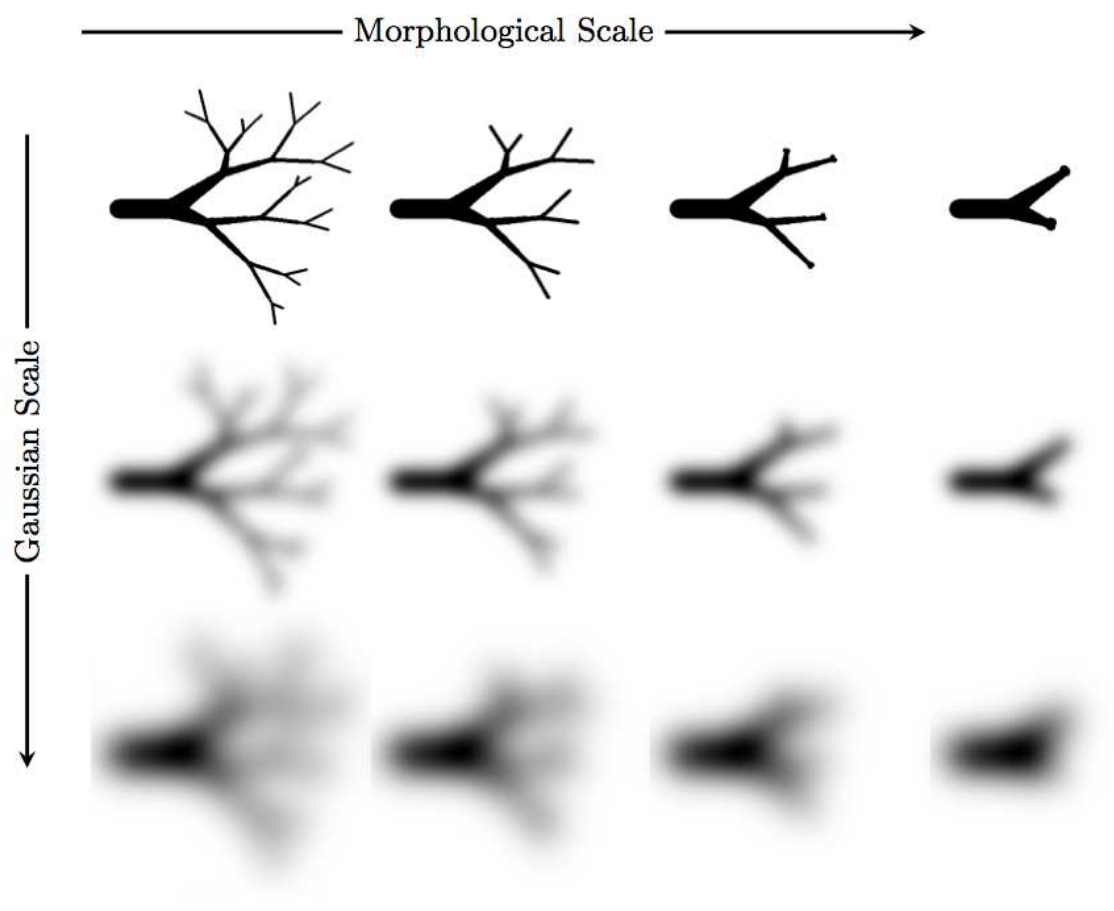

Fig. 2. 2D combined morphological and Gaussian scale 
Table 1. SSD relative to pre-registered data after non-rigid registration on the finestlevel, using the Gaussian scale-space, and using the combined morphological and Gaussian scale-space.

\begin{tabular}{lrrrrrr}
\hline Strategy & Case 1 & Case 2 & Case 3 & Case 4 & Case 5 & Case 6 \\
\hline Original & $63.95 \%$ & $36.27 \%$ & $43.90 \%$ & $52.71 \%$ & $17.16 \%$ & $34.75 \%$ \\
Gaussian & $20.59 \%$ & $23.46 \%$ & $22.70 \%$ & $27.52 \%$ & $9.21 \%$ & $24.15 \%$ \\
Morph.+Gauss. & $19.45 \%$ & $23.17 \%$ & $23.49 \%$ & $26.59 \%$ & $8.31 \%$ & $21.25 \%$ \\
\hline
\end{tabular}

\section{Results}

We tested our method on the registration of hepatic vessels obtained from 3D CT and ultrasound. We totally considered 6 cases. The size of the images was between $128^{3}$ and $256^{3}$ voxels and for each image we constructed 4 morphological and 4 Gaussian scales. The morphological scales were constructed by opening the images with balls of diameters $r=1,5,11,15$ voxels. For the Gaussian scales we used discrete convolution kernels based on the $1 \mathrm{D}$ stencil [ $\left[\begin{array}{lll}0.25 & 0.5 & 0.25\end{array}\right]$. Higher order smoothing was achieved by repeated convolution. Therefore, here the smoothing parameter $\sigma$ is a discrete number that denotes the number of subsequent convolutions. For our tests we generated 4 scales for $\sigma=0,4,8,16$.

We compared three different registration strategies. First, we used plain non-rigid registration on the finest level for $(r, \sigma)=(1,0)$. Second, we used the Gaussian scale-space and performed 4 registrations by choosing $(r, \sigma)$ as $(1,16)$, $(1,8),(1,4)$, and $(1,0)$. The third strategy uses both, the morphological and Gaussian scale. Here, we chose the sequence of $(r, \sigma)$ as $(15,16),(15,8),(11,8)$, $(11,4),(5,4),(5,0)$, and $(1,0)$.

Furthermore, for each strategy we performed rigid pre-registration.

The results are summarized in Table 1 and a visual comparison for the 4 th case is shown in Figure 3.

We found that both scale-space approaches produced good results and significantly outperformed only registration on the finest scale. Thereby, the combined morphological and Gaussian strategy produced the best results.

\section{Discussion}

We have presented a scale-space approach for the registration of tubular tree-like structures such as vessels. The key-feature is a combined two-dimensional morphological and Gaussian scale-space. The morphological axis allows for scaling the structural level and the Gaussian axis spreads local information to find a globally optimal deformation.

We tested our approach on real data and compared non-rigid registration of the initial images, using a pure Gaussian scale-space, and the combined twodimensional scale-space. Our experiments showed that the registration significantly benefits from using scale-spaces. Although, the results of the combined 
Fig. 3. Registration results for Case 4 (reference bright, template dark)

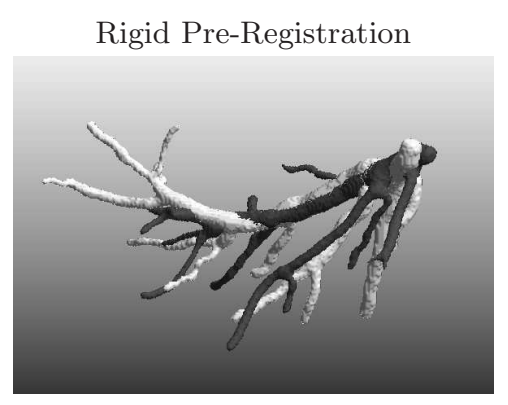

Gaussian

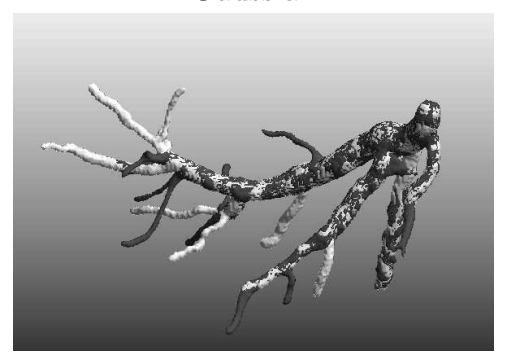

Non-Rigid

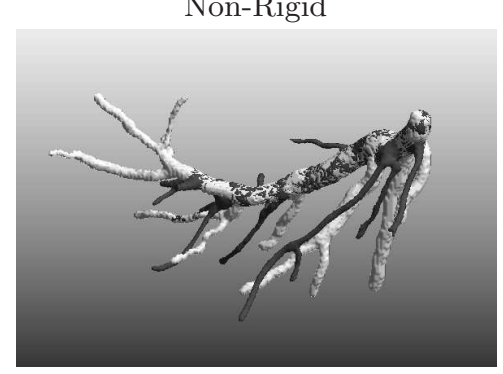

Morphological+Gaussian

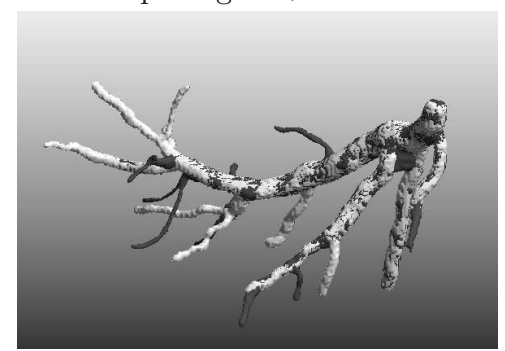

framework are best, to our surprise they are comparable to the pure Gaussian approach which needs further investigation in the future.

A practical benefit is that the method is easy to implement and due to the used scale-spaces it is robust with respect to noise and lack of data.

Summarizing, we conclude that using scale-spaces ideas is inevitable for successful registration of vessel structures.

\section{References}

1. Charnoz A, Agnus V, Malandain G, et al. Design of robust vascular tree matching: Validation on liver. LNCS. 2005;3566:443-455.

2. Reinertsen I, Descoteaux M, Siddiqi K, et al. Validation of vessel-based registration for correction of brain shift. Med Image Anal. 2007;11(4):374-388.

3. Aylward SR, Jomier J, Weeks S, et al. Registration and analysis of vascular images. Int J Computer Vis. 2003;55(2-3):123-138.

4. Bühler K, Felkel P, La Cruz A. Geometric methods for vessel visualization and quantification: A survey. In: Brunett G, Hamman B, Müller H, editors. Geometric Modelling for Scientific Visualization. Springer; 2003.

5. Lange T, Eulenstein S, Hünerbein M, et al. Vessel-based non-rigid registration of $\mathrm{MR} / \mathrm{CT}$ and 3D ultrasound for navigation in liver surgery. Comput Aided Surg. $2003 ; 8(5): 228-240$.

6. Modersitzki J. Numerical Methods for Image Registration. Numerical Mathematics and Scientific Computation. Oxford University Press; 2003.

7. Weickert J. Anisotropic Diffusion in Image Processing. Teubner; 1998. 